\title{
Supplementary Table 1: Search strategy for "The role of social determinants of health in the risk and prevention of group A streptococcal infection, acute rheumatic fever and rheumatic heart disease: A systematic review"
}

\begin{tabular}{|c|c|}
\hline Database & ategy \\
\hline $\begin{array}{l}\text { Pubmed } \\
\text { (MeSH } \\
\text { Terms) }\end{array}$ & $\begin{array}{l}\text { acute rheumatic fever[MeSH Terms] OR rheumatic heart disease[MeSH Terms] OR } \\
\text { streptococcus pyogenes[MeSH Terms] } \\
\text { AND } \\
\text { housing[MeSH Terms] OR environment and public health[MeSH Terms] OR infectious } \\
\text { disease transmission[MeSH Terms] OR social environment[MeSH Terms] OR } \\
\text { poverty[MeSH Terms] OR socioeconomic fact ORs[MeSH Terms] OR residence } \\
\text { characteristics[MeSH Terms] OR social conditions[MeSH Terms] OR sanitation[MeSH } \\
\text { Terms] OR water supply[MeSH Terms] OR life style[MeSH Terms] OR poverty areas } \\
\text { AND } \\
\text { Intervention study[MeSH Terms] OR health promotion[MeSH Terms] OR } \\
\text { epidemiology[MeSH Terms] OR population surveillance[MeSH Terms] OR primary } \\
\text { prevention[MeSH Terms] OR health education[MeSH Terms] OR community } \\
\text { survey[MeSH Terms] } \\
\text { WITH LIMITS } \\
\text { Randomized Controlled Trial; Clinical Trial; Controlled Clinical Trial; Evaluation Studies; } \\
\text { Journal Article; Meta-Analysis; Multicenter Study; Observational Study; Systematic } \\
\text { Reviews; Abstract; Humans; English }\end{array}$ \\
\hline $\begin{array}{l}\text { Pubmed } \\
\text { (Key word } \\
\text { search) }\end{array}$ & $\begin{array}{l}\text { Acute rheumatic fever OR Rheumatic heart disease OR Group A streptococcus } \\
\text { AND } \\
\text { housing OR environmental factors OR socioeconomic OR poverty OR crowding OR } \\
\text { overcrowding OR nutrition } \\
\text { WITH LIMITS: } \\
\text { Humans; English }\end{array}$ \\
\hline $\begin{array}{l}\text { Cochrane } \\
\text { library } \\
\text { (Key word } \\
\text { search) }\end{array}$ & $\begin{array}{l}\text { Acute rheumatic fever OR Rheumatic heart disease OR Group A streptococcus } \\
\text { AND } \\
\text { housing OR environmental factors OR socioeconomic OR poverty OR crowding OR } \\
\text { overcrowding OR nutrition } \\
\text { WITH LIMITS: } \\
\text { Humans; English }\end{array}$ \\
\hline $\begin{array}{l}\text { Embase } \\
\text { (Emtree } \\
\text { term } \\
\text { search) }\end{array}$ & $\begin{array}{l}\text { 'rheumatic fever'/exp OR 'rheumatic fever' OR 'rheumatic heart disease'/exp OR } \\
\text { 'rheumatic heart disease' OR 'streptococcus group a'/exp OR 'streptococcus group a' } \\
\text { AND } \\
\text { 'demography'/exp OR 'demography' OR 'disease transmission'/exp OR 'disease } \\
\text { transmission' OR 'social environment'/exp OR 'social environment' OR 'poverty'/exp }\end{array}$ \\
\hline
\end{tabular}




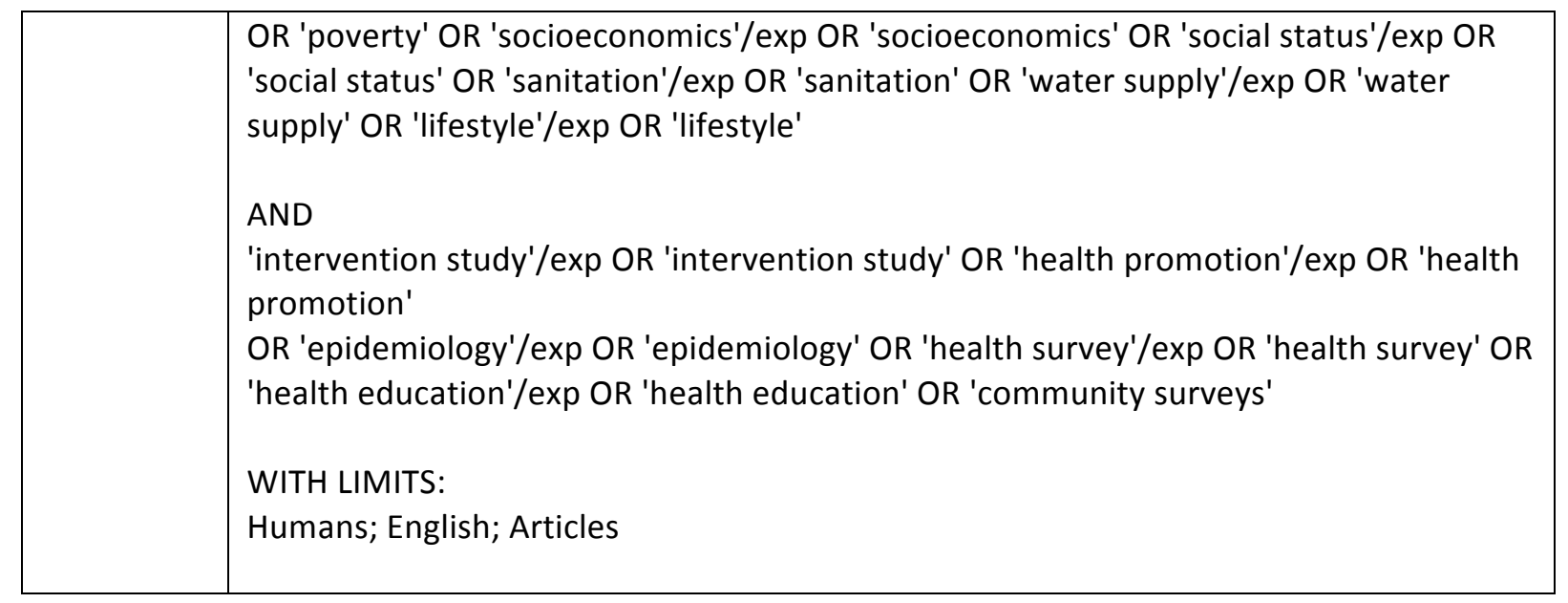

